Representation of motion

\section{boundaries in retinotopic human visual cortical areas}

\author{
John B. Reppas ${ }^{\star} \dagger$, Sourabh Niyogi $\ddagger$, Anders M. Dale $\dagger$, \\ Martin I. Serenos \& Roger B. H. Tootell $\dagger$
}

${ }^{*}$ Department of Neurobiology, Harvard Medical School, 220 Longwood Avenue, Boston, Massachusetts 02115, USA

$\dagger$ Massachusetts General Hospital Nuclear Magnetic Resonance Center, 149 13th Street, Charlestown, Massachusetts 02129, USA

$\ddagger$ Perceptual Science Group, Department of Brain and Cognitive Science, Massachusetts Institute of Technology, Cambridge, Massachusetts 02139, USA $\$$ Department of Cognitive Sciences, University of California at San Diego, La Jolla, California 92093-0515, USA

Edges are important in the interpretation of the retinal image. Although luminance edges have been studied extensively, much less is known about how or where the primate visual system detects boundaries defined by differences in surface properties such as texture, motion or binocular disparity. Here we use functional magnetic resonance imaging (fMRI) to localize human visual cortical activity related to the processing of one such higher-order edge type: motion boundaries. We describe a robust fMRI signal that is selective for motion segmentation. This boundary-specific signal is present, and retinotopically organized, within early visual areas, beginning in the primary visual cortex (area V1). Surprisingly, it is largely absent from the motionselective area MT/V5 and far extrastriate visual areas. Changes in the surface velocity defining the motion boundaries affect the strength of the fMRI signal. In parallel psychophysical experiments, the perceptual salience of the boundaries shows a similar dependence on surface velocity. These results demonstrate that information for segmenting scenes by relative motion is represented as early as V1.

Clinical and psychophysical evidence suggests that humans possess specialized mechanisms for detecting motion boundaries ${ }^{1,2}$. However, it is unclear where boundary detection occurs in the visual pathway. The observation that motion segmentation is rapid and pre-attentive ${ }^{3}$ suggests an early visual mechanism, and there is some neurophysiological evidence for this ${ }^{4}$. At the same time, boundary mechanisms also integrate information over a large region of visual space and a range of motion coherences ${ }^{5,6}$. These latter properties are consistent with an extrastriate detection mechanism-a view supported by theoretical considerations ${ }^{7}$ and by preliminary human brain-mapping results ${ }^{8,9}$. Here we test whether an initial motionsensing stage in early visual areas (for example, V1, V2, MT) precedes motion boundary detection by later areas in the human dorsal or ventral visual processing streams.

The stimulus was a full-field random dot texture pattern that alternated between periods of segmented and uniform motions. In both the uniform and segmented conditions, the direction of local motion reversed every $1.2 \mathrm{~Hz}$. Thus, the time-averaged local image statistics (contrast, spatial pattern, direction of motion) were identical for the segmented and uniform portions of the scan. The two conditions were distinguished only by the presence or absence of motion boundaries. We used boundaries created in three ways: (1) by shearing motions, in which local motions were parallel to the motion edge; (2) by compressive motions, in which local motions were perpendicular to the edge; and (3) by a checkerboard boundary pattern containing both types of edges. The default texture velocity was 5 degrees per second. This velocity produced the most visible boundaries in our displays, and is typical of velocities used in perceptual studies of motion segmentation ${ }^{6,9}$. a

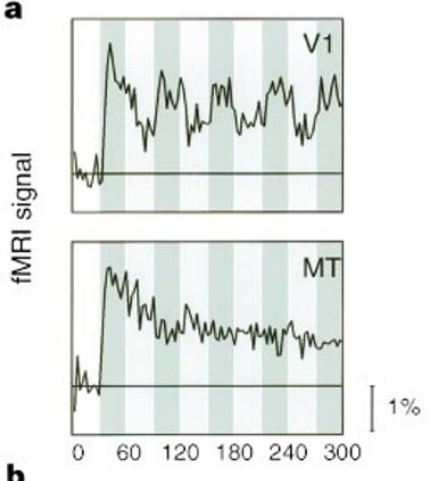

b

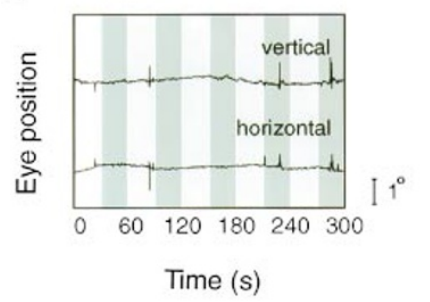

Figure 1 The motion segmentation fMRI signal and its anatomical distribution. The subject viewed a uniform motion (light green shading) alternating every $30 \mathrm{~s}$ with a checkerboard pattern formed by relative motion (darker shading). One 'check' subtended $\sim 0.9$ by 0.9 degrees. For the first 30 s, the display was blank except for the fixation point. a, The fMRI signal changes in V1 and MT/V5 are expressed as a percentage change from the signal during this baseline period. $\mathbf{b}$, Horizontal and vertical positions of the right eye; shading as in $\mathbf{a}$. Transients correspond to blinks. c, Areas selectively activated during the motion boundary portions of the scan. Dotted blue and green lines on the medial and lateral views of the inflated hemisphere indicate the 'cuts' needed to flatten the occipital lobe. Gyri and sulci are indicated in light and dark grey, respectively. Solid and dotted white lines indicate the representation of the horizontal and vertical meridia respectively (see Methods). Scale bar, $1 \mathrm{~cm}$.

Moreover, it is well matched to the velocity selectivity reported for opponent motion neurons in the non-human primate ${ }^{10}$. Subjects were instructed to fixate a central cross-hair and view the stimulus passively. In some cases, eye movements were monitored to document the stability of fixation.

Compared to a baseline of mean luminance, the response to the experimental stimulus had two components in area V1 and the other retinotopic areas: (1) a constant elevation of the fMRI signal beginning at the onset of the stimulus and lasting the entire experiment; and (2) a stimulus-locked response superimposed on the constant response (Fig. 1a). The constant component represents the response to the visual stimulus elements that are present throughout the experiment, namely the visual texture and its reversing motion. The stimulus-locked component originates from mechanisms that are selectively activated during segmented but not uniform motion. Eye movements were not significantly different during segmented versus uniform motion periods (Fig. $1 b)$, suggesting that the modulated fMRI signal seen in the retinotopic visual areas is due to interactions of the stimulus with purely sensory mechanisms. In area MT/V5 and far extrastriate visual areas, the fMRI response typically consisted only of the sustained component. We never observed an elevated response for uniform over segmented motions in any part of the visual cortex. The fMRI signals did not depend critically on the type of motion boundary used, whether shearing, compressive or both.

The motion segmentation signal was localized on the flattened visual cortices of 6 subjects ( 12 hemispheres). In each subject we also mapped the boundaries of the overtly retinotopic visual areas 
(V1, V2, V3/VP, V3A and V4v) using phase-encoded retinotopic stimulation $^{11-13}$. Area MT/V5 was located using a low-contrast radial motion stimulus ${ }^{14}$. Figure $1 \mathrm{c}$ shows the response to a motion-boundary defined checkerboard pattern in a representative subject relative to the retinotopic visual areas and MT/V5. For the high density of motion boundaries in this stimulus, the selective response to motion boundaries is strongest in V1 and V2, present in $\mathrm{V} 3$ and VP and largely absent in V3A and V4v. Strong fMRI signals selective for motion boundaries were not observed in MT/V5 of this subject, nor in those regions of the occipital, temporal and parietal lobes which are thought to contain the human homologues of macaque dorsal and ventral visual streams ${ }^{15}$. Similar results were obtained in the five other subjects.

When the number of motion boundaries in the stimulus was reduced, we observed that the segmentation signal within the early visual areas became patchy, suggestive of discrete responses to individual boundaries within the visual field. To test this hypothesis, we presented a single motion boundary in a region of the visual field that could be identified easily using an independent retinotopic criterion. The stimulus was a rotating texture pattern which alternated between a uniform motion and a shearing motion that created a single, stationary, circular boundary located $\sim 7$ degrees eccentric to the fixation point (Fig. 2a). The representation of this circle in visual space was independently confirmed from isoeccentricity phase maps in the same subjects ${ }^{12}$.

The circular motion boundary produced a modulated response in two distinct regions (Fig. 2a). These occur in the medial bank of the occipital lobe and are oriented perpendicular to the boundaries between retinotopic visual areas. The stripes of activation are limited to V1, V2 and V3/VP and occur at the representation of the 7-deg isoeccentricity line. The spatial extent of the boundary activation is comparable to that produced by a thin ring of counterphasing checkerboard in the same position. This suggests that the $\mathrm{V} 1 / \mathrm{V} 2 / \mathrm{V} 3$ response to motion boundaries engages a detection mechanism of similar dimensions to that of luminance. Similar results were obtained with stationary wedge boundaries formed by compressive dot motions. If the single boundary moved through the visual field during the segmented epochs, the localized modulation of the fMRI signal was no longer observed. This result demonstrates that motion boundaries not only activate early visual areas, but that they do so in a retinotopically specific fashion.

We examined the strength of the fMRI segmentation signal as the number of boundaries in the display was systematically varied. The stimulus for this experiment alternated between a rotating uniform motion and a pinwheel pattern formed by compressive motions (5\% dot density). The pinwheel pattern was chosen because texture velocity and boundary size both vary with eccentricity. Because motion-defined boundaries are detected by a localized retinotopic mechanism (Fig. 2), the motion edges were rotated with the carrier motion to engage detection mechanisms at all spatial locations. Five subjects were scanned using the standard velocity of 5 degrees per second, where the boundaries are most visible (Fig. 4). The tuning curves were repeated in two subjects at a higher velocity (30 degrees per second), better matched to the median velocity selectivity of MT cells $^{16}$. We wanted to eliminate the possibility that the lack of a modulated signal in MT/V5 simply reflected the relatively low velocity of the standard carrier motion. At these high carrier velocities, subjects report not seeing any motion boundaries (Fig. 4c).

Figure 3 summarizes how the segmentation fMRI signal varied with the number of boundaries. For the retinotopic areas, the curves have an inverted ' $U$ ' shape: the modulated response is low for sparse and dense boundary configurations, and maximal for an intermediate density. The peaks of these tuning curves are different for each retinotopic area. V1, which represents one extreme of the response, is optimally stimulated by motions that are segmented at a fine scale (16-32 wedges in the display), whereas V3A, at the other extreme, is best stimulated by coarser motion segmentation (4-8 wedges in the display). The peaks of areas V2 and V3/VP are intermediate between V1 and V3A (results not shown). These results suggest that spatial scale is an important determinant of the fMRI response to segmented motions, and that one function of the retinotopic areas may be to segment moving images at different scales $^{17}$. Data from MT/V5 show that it is much less selectively activated than the retinotopic areas over the entire range of boundary densities, for both high and low velocity displays. When stationary boundaries were used, the results were similar (data not shown). For dense texture displays, the tuning curves extended to higher boundary densities. Unlike single units in macaque MT, no suppression of the MT/V5 fMRI signal was observed for transparent motions ${ }^{18}$, but the stimulus and physiological methods in the present experiments differed from those previous experiments.

The response curve for each retinotopic area falls off at boundary densities when subjects reported no longer seeing distinct kinetic edges, but rather a display that approximated transparent motion ${ }^{19}$.
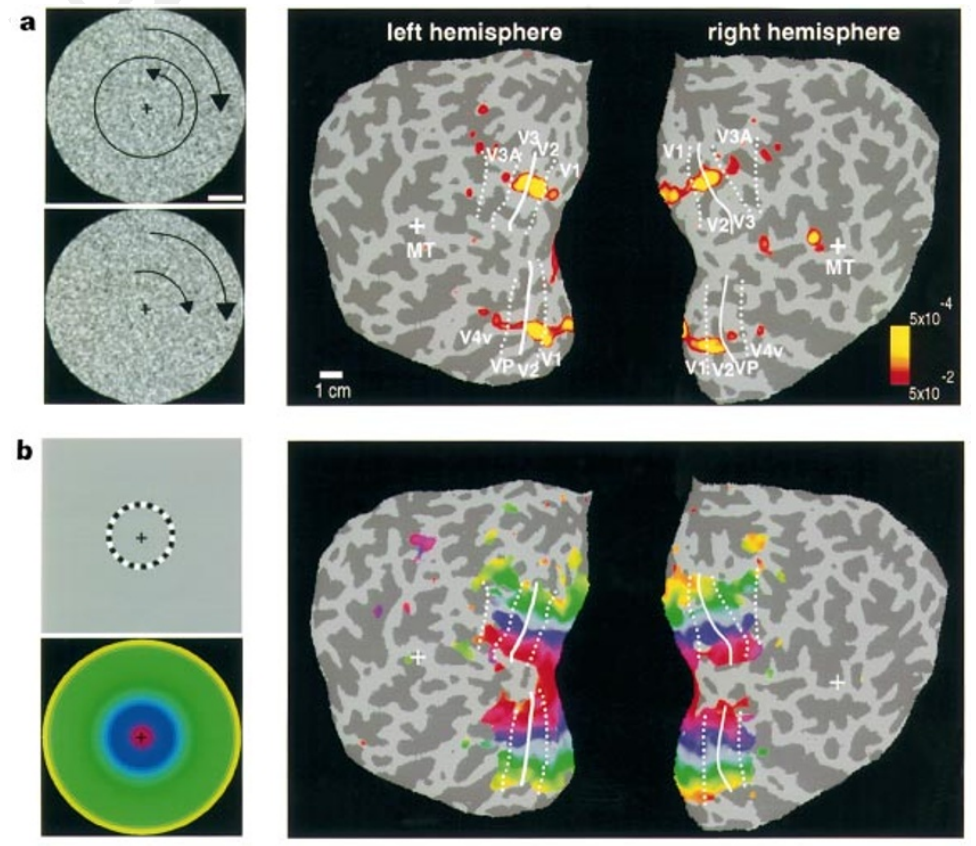

Figure $2 \mathrm{~A}$ circular motion boundary produces modulated fMRI activity at the cortical representation of that circle. a, Left, the subject viewed a single circular motion boundary about 7 deg eccentric to the fixation point which alternated with uniform motion; a single frame of this texture is shown (scale bar, $5 \mathrm{deg}$ ). Right, the yellow to red pseudocolour indicates the distribution of stimulus-locked modulation in the fMRI signal in the left and right flattened hemispheres. The borders of the visual areas are as in Fig. 1. b. Isoeccentricity maps ${ }^{12}$ for a thin checkerboard ring (top left panel) which expanded repeatedly from the fixation point to the visual periphery. Pseudocolour (right) indicates eccentricity in the visual field as determined by phaseencoded retinotopic mapping. The pattern of localized activity in a corresponds to a region of constant eccentricity in panel $\mathbf{b}$. Scale bar, $1 \mathrm{~cm}$. 
In fact, we observed no selective fMRI modulation in experiments of transparent versus uniform motions (Fig. 3). This finding provides an important control for the hypothesis that the FMRI signal we observed is a bona fide boundary signal. The presence of two directions of motion in the same part of the visual field, which is the case for both motion boundaries and motion transparency, is not sufficient to generate a selective fMRI response. A perceptual boundary must be present.

To explore further the origin of the modulated fMRI signal, we examined how motion boundaries formed by different surface velocities affect the motion segmentation signal. Boundaries formed by two very slow or two very fast motions are harder to see than those formed by two intermediate motion velocities, at least for brief stimulus presentation ${ }^{20,21}$. If the modulated response we observe originates from a boundary detection mechanism, it should be: (1) related to the subjective experience of motion boundaries over a range of boundary visibility; and (2) dissociable from a pure visual motion signal. The fMRI segmentation signal in V1 was measured for different surface velocities with a boundary density $(\sim 0.5$ cycles per degree (c.p.d.)) optimized for V1 fMRI responses in four subjects. In separate tests, we measured the visibility of these boundaries as a function of surface velocity using a two-alternative forced-choice task in which subjects were instructed to discriminate segmented from uniform motions.

The modulated component of the V1 fMRI signal is maximal at an intermediate velocity of $\sim 5$ degrees per second, and decreases for higher and lower velocities (Fig. 4a). Because the sustained component of the fMRI signal does not vary with stimulus velocity, this variation in the strength of the segmentation signal cannot be explained by the failure of V1 to register fast and slow motions
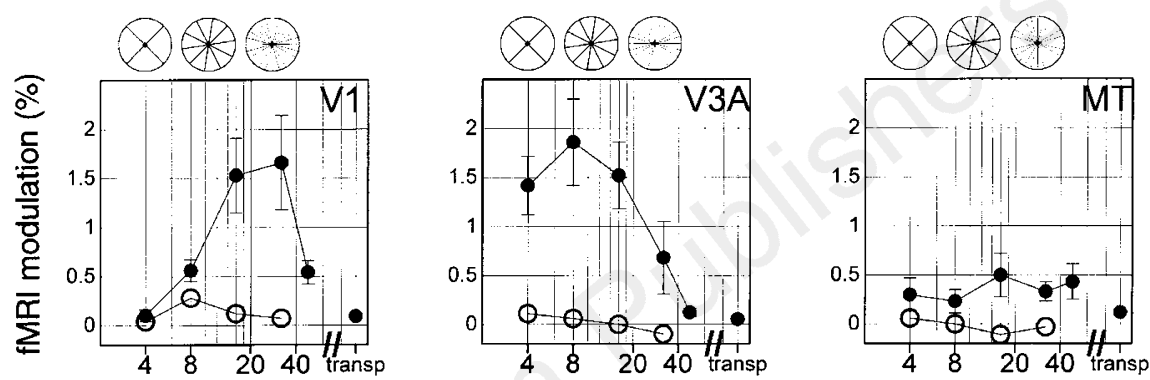

Number of motion boundaries in the display

Figure 3 Tuning of the $\mathrm{fMRI}$ segmentation signal in three visual areas as a function of boundary density. The stimulus was a rotating texture (dot density, 5\%) that alternated every $30 \mathrm{~s}$ between uniform rotational motion and pinwheel boundaries formed by compressive motions. Wedge angle varied from 90 deg to 7.5 deg of arc. We measured the strength of the fMRI segmentation signal as the number of wedges in the stimulus was varied. A given density was shown 1-3 times in a randomly interleaved fashion, and the results were averaged for each density tested. Filled points represent the mean \pm s.d. percentage modulation of the $\mathrm{fMRI}$ signal for 5 subjects at $5 \mathrm{deg} \mathrm{s}^{-1}$. Open points are the mean percentage modulation for 2 subjects at $30 \mathrm{deg} \mathrm{s}^{-1}$. At low velocity, retinotopic areas showed robust responses to the boundary stimulus and were selective for a range of boundary densities. However, when transparent motion was alternated with uniform motion, they showed no selective activation. MT/N5's response to boundaries was less prominent and not tuned for boundary density at either velocity.

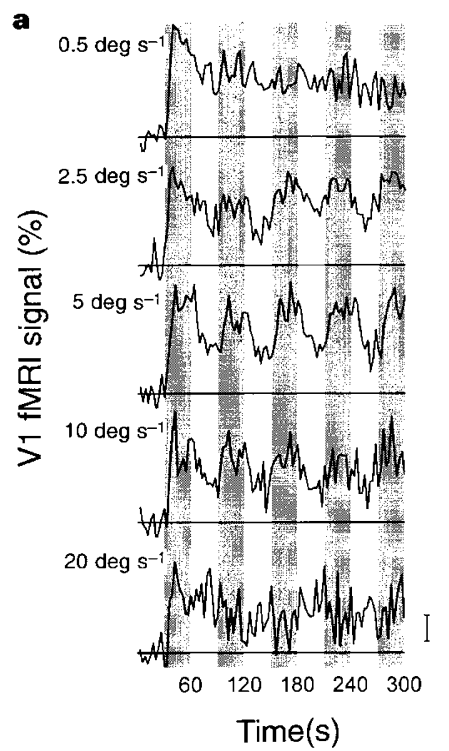

Figure 4 The modulated component of the $\mathrm{fMRI}$ segmentation signal varies with the perceptual salience of the motion boundary display. The stimulus, optimized for area $V 1$, contained a fixed number of concentric circular boundaries ( $\sim 0.5$ c.p.d.) formed by shearing motion of the dense texture. These alternated with uniform rotational motion every $30 \mathrm{~s}$. In successive scans, the velocity of the carrier motion was varied. Each of the six velocities was presented 1-3 times per scanning session in a randomly interleaved fashion. a, Raw time-courses for 5
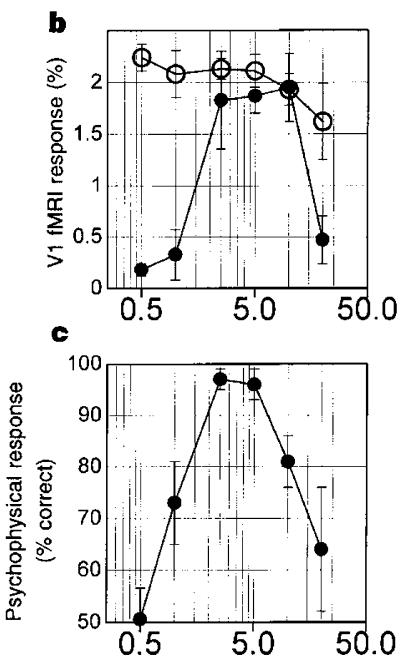

Surface velocity $\left(\operatorname{deg~s}^{-1}\right)$ velocities, sampled from V1 of one subject. Shading indicates periods of segmented motion. Scale bar, 1\% signal change. b. Averaged strength of both the modulated (black circles) and steady-state (white circles) signal change relative to the mean luminance baseline (mean \pm s.d.; $n=4$ ). c. Mean performance on a psychophysical task that required subjects to distinguish segmented from uniform motion (mean \pm s.d.; $n=3$ ). 
per se (Fig. 4b). Rather, the average modulated fMRI response is related to the visibility of the boundaries, expressed as average performance in a standard boundary detection task ${ }^{21}$. As subjects see the boundaries less distinctly, the fMRI signal in V1 modulates less and less in response to the stimulus (Fig. 4c). The duration of the stimuli used in the fMRI experiment is much longer than that used for psychophysical detection experiments, and at these longer durations the boundary is easily perceived at all velocities tested. Thus the fMRI signal does not correlate directly with perception. Nevertheless, the similarity in the effects of velocity in the two experiments (compare Fig. 4b,c) suggests that the fMRI signal reflects processes within V1 that are likely also to be relevant to perception of motion boundaries. The dissociation between the fMRI response to the boundaries themselves versus the motions that define the boundaries suggests that the response properties of motion boundary mechanisms in V1 differ from the betterknown striate cortical mechanisms that register stimulus pattern and motion.

Our results are inconsistent with the views that: (1) neurons in early visual areas (V1, V2, V3) register only the local direction of image motion; and (2) selectivity for higher-order optic flow patterns, like motion boundaries, is developed only late in the motion processing pathway (for example, MT and MST) ${ }^{6}$. The fMRI responses we describe may originate from boundary-sensitive mechanisms within the retinotopic areas, possibly involving longrange horizontal connections ${ }^{4}$. Alternatively, feedback from extrastriate motion areas may contribute to the segmentation responses seen in retinotopic areas ${ }^{22}$.

Our findings do not preclude the contribution of non-retinotopic cortical areas, and area MT/V5 in particular, to the detection of motion boundaries. The lack of a strongly selective motion segmentation signal from MT/V5 over a range of boundary densities and surface velocities might simply result from the presence of approximately equal numbers of wide-field and opponent-motion neurons ${ }^{23}$. Alternatively, human MT/V5 may simply not be important for motion segmentation, consistent with a previous PET study ${ }^{9}$, neuropsychological findings ${ }^{5}$, and experimental results in the macaque $e^{24,25}$. Certainly one important role of monkey MT appears to be the integration of local motion signals across space and time to form global representations of motion $^{26}$ - a process that may 'blur' motion boundary information.

\section{Methods}

Experimental design. All imaging experiments compared fMRI signals during a motion boundary condition to those produced during a uniform motion condition. These conditions alternated either every $16 \mathrm{~s}$ (Figs $1 \mathrm{c}, 2$ ) or every $30 \mathrm{~s}$ (Figs 1a, 3, 4). The order of segmented and uniform conditions varied. Boundaries were not visible in the absence of display motion, and single stimulus frames from the boundary condition contained no significant Fourier energy at the orientations and bandwidths of the motion boundary pattern. We used two types of random dot stimuli: a dense version, in which each pixel was assigned to one of 255 grey values, and a sparse pattern of white dots on a black background (dot size $\sim 0.15 \times 0.15 \mathrm{deg}$; overall dot density was $5 \%$ ). The dot velocity was $\sim 5 \mathrm{deg} \mathrm{s}^{-1}$ unless stated otherwise; this is the display velocity at which boundaries are most visible (Fig. 4). The stimuli were generated on a Silicon Graphics Onyx computer using GL graphics functions at a frame rate of $60 \mathrm{~Hz}$. The output of the graphics window was sent to a Sony 2000 LCD projector, which projected images into the bore of the magnet, where they subtended $\sim 26$ by 26 degrees. Software control of screen intensity was calibrated using a Spectraspot photometer (Photoresearch). Eye position was recorded using an infrared monitoring device (Ober2, Sweden) and sampled at $10 \mathrm{~Hz}$. Linear drift of the eye position traces over the recording period was subtracted. In all, 16 subjects were scanned with motion boundary stimuli. Of these, six were studied in detail using cortical flat mapping techniques, six more contributed to tuning curves (Figs 3,4), and data from the remainder were used to confirm the basic findings. Most subjects used rigidly mounted, individually moulded, deep-impression bite bars. Head motion did not affect scans that contributed to the flat maps and tuning curves shown, presumably reflecting the constraints of the bite bar and the previous fMRI experience of these subjects.

MRI scans. Subjects were scanned on a 1.5 T GE magnet, retrofitted for echoplanar imaging (Advanced NMR), using a bilateral quadrature surface coil overlying the occipital, inferior parietal and posterior temporal cortex. In functional imaging experiments, we used an asymmetric spin-echo sequence (TR $=2,000-4,000 \mathrm{~ms}, \mathrm{TE}=70 \mathrm{~ms}$, with a $180 \mathrm{deg}$ refocusing pulse offset of $-25 \mathrm{~ms}$ ), to visualize the BOLD (blood oxygen level-dependent) contrast changes that accompany brain activity ${ }^{14,27-29}$. Images were acquired in 14-16 contiguous 4-mm slices oriented approximately perpendicular to the calcarine fissure; in-plane resolution was $3 \mathrm{~mm} \times 3 \mathrm{~mm}$. Statistical maps of the modulated fMRI signal were constructed by performing a fast-Fourier transform on each voxel's time-course and performing an F-test on the ratio of stimuluslocked power to all other non-harmonic frequencies, for frequencies above 3 cycles per scan. The resulting $P$ values (null hypothesis: no stimulus-locked modulation) were smoothed using 10 iterations of a box-car filter and thresholded. Three-dimensional reconstructions of the subjects' brains were 'inflated' by an iterative algorithm that relaxed and minimized angular and areal distortion ${ }^{30}$. Flattened views of the occipital lobe were obtained by removing the occipital region from the inflated hemispheres, cutting it open along the fundus of the calcarine fissure, and allowing it to unfold ${ }^{12}$. The boundaries between the overtly retinotopic areas were determined by transitions in the visual field sign map, which is derived from a combination of eccentricity and polar angle phase maps ${ }^{11-13}$. Here, the representations of the vertical meridian are dotted, and the horizontal meridian are solid. MT/V5 was localized by its selective response to a moving versus stationary concentric ring pattern of low $(2-3 \%)$ contrast $^{14}$. The boundaries between retinotopic areas and the centroid of the MT activation were overlaid onto maps of the motion segmentation fMRI signal. For those experiments in which we acquired timecourse data, all voxels that were $2-3 \mathrm{~mm}$ (one voxel) within the boundaries of each of the labelled areas and which were not obviously confounded by macrovascular artefact (high amplitude/frequency in the time course, and/or location coextensive with large vessels in the angiogram) were selected and averaged together ${ }^{14}$. Stimulus-evoked modulation is expressed as the percentage excursion from the signal in the unstimulated condition.

Psychophysics. We used a temporal two-alternative forced choice task requiring subjects to distinguish between the concentric motion boundaries used in the fMRI experiments (Fig. 4) and a uniform motion at different carrier speeds. Each trial began with a blank screen with a central fixation point, and contained two 100-ms stimulus presentations (one segmented and one uniform condition) separated by $\sim 1 \mathrm{~s}$ (ref. 21 ). The order of the segmented and uniform motion conditions, the directions of the uniform motion and the overall stimulus speed were all randomly varied from trial to trial. Subjects were instructed to indicate whether the boundary stimulus came first or second in the trial, and to guess when unsure. Performance was assessed by the percentage of correct responses (30 trials per velocity).

Received 19 December 1996; accepted 30 April 1997.

1. Braddick, O. A short-range process in apparent motion. Vision Res. 14, 519-527 (1974).

2. Regan, D., Giaschi, D., Sharpe, J. A. \& Hong, X. H. Visual processing of motion-defined form: selective failure in patients with parietotemporal lesions. J. Neurosci. 12, 2198-2210 (1992).

3. Dick, M., Ullman, S. \& Sagi, D. Parallel and serial processes in motion detection. Science 237, 400-402 (1987).

4. Lamme, V. A., van Dijk, B. W. \& Spekreijse, H. Contour from motion processing occurs in primary visual cortex. Nature 363, 541-543 (1993).

5. Vaina, L. M., Grzywacz, N. M. \& Kikinis, R. Segregation of computations underlying perception of motion discontinuity and coherence. Neuroreport 5, 2289-2294 (1994).

6. Murakami, I. \& Shimojo, S. Modulation of motion aftereffect by surround motion and its dependence on stimulus size and eccentricity. Vision Res. 35, 1835-1844 (1995).

7. Nakayama, K. \& Loomis, J. M. Optimal velocity patterns, velocity-sensitive neurons, and space perception: a hypothesis. Perception 3, 63-80 (1974).

8. Patzwahl, D. R., Zanker, J. M. \& Altenmuller, E. O. Cortical potentials reflecting motion processing in humans. Vis. Neurosci. 11, 1135-1147 (1994).

9. Orban, G. A. et al. A motion area in human visual cortex. Proc. Natl Acad. Sci. USA 92, 993-997 (1995)

10. Lagae, L., Gulyas, S., Raiguel, S. \& Orban, G. A. Laminar analysis of motion information processing in macaque V5. Brain Res. 496, 361-367 (1989).

11. Engel, S. A. et al. fMRI of human visual cortex. Nature 369, 525 (1994).

12. Sereno, M. I. et al. Borders of multiple visual areas in humans revealed by functional magnetic resonance imaging. Science 268, 889-893 (1995).

13. DeYoe, E. A. et al. Mapping striate and extrastriate visual areas in human cerebral cortex. Proc. Natl Acad. Sci. USA 93, 2382-2386 (1996).

14. Tootell, R. B. H. et al. Functional analysis of human MT and related visual cortical areas using magnetic resonance imaging. J. Neurosci. 15, 3215-3230 (1995). 
15. Reppas, J. B., Dale, A. M., Sereno, M. I. \& Tootell, R. B. H. La vision: une perception subjective. $L a$ Recherche 289, 52-56 (1996).

16. Maunsell, J. H. R. \& van Essen, D. C. Functional properties of neurons in middle temporal visual area of the macaque monkey. I. Selectivity for stimulus direction, speed, and orientation. J. Neurophysiol. 49, 1127-1147 (1983).

17. Braddick, O. Segmentation versus integration in visual motion processing. Trends Neurosci. 16, 263 268 (1993)

18. Snowden, R. J., Treue, S., Erickson, R. E. \& Andersen, R. A. The response of area MT and V1 neurons to transparent motion. J. Neurosci. 11, 2768-2785 (1991).

19. van Doorn, A. J. \& Koenderink, J. J. Spatial properties of the visual detectability of moving spatial white noise. Exp. Brain Res. 45, 189-195 (1982).

20. Banton, T. \& Levi, D. M. The perceived strength of motion-defined edges. Perception 22, 1195-1204 (1993).

21. Sachtler, W. L. \& Zaidi, Q. Visual processing of motion boundaries Vision Res. 35, 807-826 (1995). 22. Maunsell, J. H. R. \& van Essen, D. C. The connections of the middle temporal visual area (MT) and their relationship to a cortical hierarchy in the macaque monkey. J. Neurosci. 3, 2436-2586 (1983).

23. Born, R. T. \& Tootell, R. B. H. Segregation of global and local motion processing in primate middle temporal visual area. Nature 357, 497-499 (1992).

24. Schiller, P. H. The effects of V4 and middle temporal (MT) area lesions on visual performance in the rhesus monkey. Vis. Neurosci. 10, 717-746 (1993).

25. Marcar, V. L., Xiao, D. K., Raiguel, S. E., Maes, H. \& Orban, G. A. Processing of kinetically defined boundaries in the cortical motion area MT of the macaque monkey. J. Neurophysiol. 74, 1258-1270 (1995).

26. Britten, K. H., Shadlen, M. N., Newsome, W. T. \& Movshon, J. A. Responses of neurons in macaque MT to stochastic motion signals. Vis. Neurosci. 10, 1157-1169 (1993).

27. Kwong, K. K. et al. Dynamic magnetic resonance imaging of human brain activity during primary sensory stimulation. Proc. Natl Acad. Sci. USA 89, 5675-5679 (1992).

28. Ogawa, S. et al. Intrinsic signal changes accompanying sensory stimulation: functional brain mappin with magnetic resonance imaging. Proc. Natl Acad. Sci. USA 89, 5951-5955 (1992).

29. DeYoe, E. A., Bandettini, P., Neitz, J., Miller, D. \& Winans, P. Functional magnetic resonance imaging (fMRI) of the human brain. J. Neurosci. Meth. 54, 171-187 (1994).

30. Dale, A. M. \& Sereno, M. I. Improved localization of cortical activity by combining EEG and MEG with MRI cortical surface reconstruction: A linear approach. J. Cogn. Neurosci. 5, 162-176 (1993).

Acknowledgements. We thank E. Adelson, S. Brown, M. Livingstone and W. van Duffel for comments on earlier versions of this manuscript. S. Macknik kindly loaned us the eye-tracking device used in some experiments. We are grateful for the technical support of T. Campbell, T. Davis, M. Foley and M. Vevea, and to all of our subjects. J.B.R. was supported by an HHMI predoctoral fellowship, and R.B.H.T. by the Human Frontiers program.

Correspondence and requests for materials should be addressed to J.B.R. at Harvard Medical Schoo (e-mail:jreppas@nmr.mgh.harvard.edu).

\section{The synaptic activation of kainate receptors}

\section{Michel Vignes \& Graham L. Collingridge}

Department of Anatomy, School of Medical Sciences, University of Bristol, University Walk, Bristol BS8 1TD, UK

L-Glutamate, the principal excitatory neurotransmitter in the vertebrate central nervous system, acts on three classes of ionotropic glutamate receptors, named after the agonists AMPA, NMDA and kainate ${ }^{1}$. AMPA receptors are known to mediate fast synaptic responses and NMDA receptors to mediate slow synaptic responses at most excitatory synapses in the brain ${ }^{2}$. Kainate receptors are formed from a separate set of genes (GluR5-7, KA-1 and KA-2) and are widely distributed throughout the brain $^{3-8}$. They are implicated in epileptogenesis and cell death'. However, the physiological functions of kainate receptors are not known $^{7}$. The development of 2,3-benzodiazepine antagonists that are selective for AMPA receptors ${ }^{10}$ enables kainate receptors to be specifically activated by exogenous ligands, such as kainate ${ }^{11-16}$. Here we demonstrate that high-frequency stimulation of mossy fibres in rat hippocampal slices, in the presence of the highly selective AMPA receptor antagonist GYKI 53655 (refs 13-15) plus NMDA- and GABA-receptor antagonists, activates an inward current in CA3 neurons that has a pharmacology typical of kainate receptors. The finding that kainate receptors can be activated synaptically adds to the diversity of information transfer at glutamatergic synapses.

Whole-cell recordings were obtained from CA3 neurons in response to single shock stimulation of the mossy fibre pathway and the associational/commissural pathway ${ }^{17,18}$ (Fig. 1a). In both pathways, AMPA-receptor-mediated excitatory postsynaptic currents (EPSCs) were blocked by the addition of GYKI 53655
$(50 \mu \mathrm{M})$, NMDA-receptor-mediated EPSCs were blocked by the selective antagonist AP5 $(100 \mu \mathrm{M})$ plus L-689,560 $(5 \mu \mathrm{M}), \mathrm{GABA}_{\mathrm{A}^{-}}$ receptor-mediated inhibitory postsynaptic currents (IPSCs) were blocked by picrotoxin $(50 \mu \mathrm{M})$ plus bicuculline $(10 \mu \mathrm{M})$, and $\mathrm{GABA}_{\mathrm{B}}$ receptor-mediated IPSCs were eliminated by the use of $\mathrm{Cs}^{+}$-based intracellular solution. In the presence of this antagonist cocktail, essentially no synaptically evoked response remained in either pathway (Fig. 1b). However, high-frequency stimulation of the mossy fibre pathway (for example, 20 shocks at $100 \mathrm{~Hz}$ ), using the same stimulus intensity, evoked a synaptic current, whereas equivalent stimulation of the associational/commissural input evoked little or no response (Fig. 1b). The size of the mossy fibreevoked EPSC was dependent on the number of stimuli delivered. The synaptic response was clearly visible following paired stimuli and increased nonlinearly in response to between two and five stimuli. The maximum peak response was elicited by 10 shocks (Fig. $2 \mathrm{a})$. The synaptic response had a linear $I-V$ relationship and a reversal potential close to $0 \mathrm{mV}(+6 \pm 2 \mathrm{mV} ; n=7)$ (Fig. $2 \mathrm{~b})$.

GYKI 53655 is a potent AMPA-receptor antagonist, which, at a concentration of $50 \mu \mathrm{M}$, does not significantly affect kainate receptors $^{13-15}$. The broad-spectrum ionotropic glutamate-receptor antagonist CNQX antagonizes kainate responses ${ }^{16,19}$, and so can be used as a kainate antagonist under conditions where AMPA and NMDA receptors are already blocked. To test the effectiveness of CNQX as a kainate receptor antagonist on CA3 neurons, its ability to reverse kainate-induced currents was tested in the presence of the antagonist cocktail $(n=8$, including $1 \mu \mathrm{M}$ tetrodotoxin in three experiments). Under these conditions, CNQX $(10 \mu \mathrm{M})$ rapidly and reversibly antagonized inward currents induced by kainate $(200 \mathrm{nM})$ (Fig. 3a). Similarly, CNQX $(10 \mu \mathrm{M})$ also rapidly and reversibly antagonized the mossy fibre-evoked synaptic current ( $n=7$; Fig. 3b). At a concentration of $20 \mu \mathrm{M}$, CNQX reduced the mossy fibre-evoked synaptic current by between 75 and $95 \%(n=7$; Fig. 3b, c).

It is unlikely that the mossy fibre-evoked response was a residual AMPA receptor-mediated response that appeared during highfrequency stimulation for the following reasons. First, GYKI 53655 was used at a concentration of approximately 50 times its half-maximal inhibitory concentration $\left(\mathrm{IC}_{50}\right)$ for antagonism at AMPA receptors ${ }^{13-15}$, and is a non-competitive antagonist, so it should be independent of L-glutamate concentration. Second, the stimulus intensities were adjusted to evoke AMPA receptormediated EPSCs of a similar size in response to associational/ commissural and mossy fibre stimulation, before the addition of the GYKI 53655-containing cocktail. A residual AMPA receptormediated EPSC should therefore have been as large after stimulation of the associational/commissural input. Third, CNQX was less potent and more rapidly reversible as an antagonist of the mossy fibre response than of an AMPA receptor-mediated EPSC recorded in brain slices. This difference correlates with the weaker potency of CNQX as a kainate as against an AMPA receptor antagonist ${ }^{16,19}$. As a further test to distinguish between AMPA and kainate receptors we applied the AMPA receptor specific potentiator cyclothiazide ${ }^{20}$. Before addition of the cocktail, cyclothiazide $(3 \mu \mathrm{M})$ produced the characteristic potentiation of a synaptic AMPA receptormediated EPSC $^{21}$ in both the associational/commissural pathway (data not shown) and the mossy fibre pathway (Fig. 4a; $n=5$ ). In contrast, when cyclothiazide was applied for the first time after addition of the cocktail, the mossy fibre-evoked EPSC was unaffected (Fig. $4 \mathrm{~b} ; n=4$ ). Activation of a residual NMDA receptormediated current can be excluded for several reasons. First, the mossy fibre-evoked synaptic current had a linear $I-V$ relationship. Second, at the concentration used $(10 \mu \mathrm{M})$, CNQX does not antagonize NMDA receptor-mediated synaptic responses in hippocampal slices. Third, a much more potent glycine site antagonist than CNQX (L-689,560) was included in the cocktail.

It has been reported that stimulation of afferent fibres can evoke a 